\title{
INFORMAÇÃO TECNOLÓGICA: IDENTIFICANDO TECNOLOGIAS, VANTAGENS E APLICAÇÕES ATRAVÉS DO BANCO NACIONAL E INTERNACIONAL DE PATENTES
}

\section{C. S. Silva', J. L. Kovaleski², S. Gaia² e A. C. Francisco ${ }^{2}$}

${ }^{1}$ Departamento de Engenharia de Produção - Universidade Federal do Rio Grande do Sul (UFRGS)

2 Departamento de Engenharia de Produção - Universidade Tecnológica Federal do Paraná (UTFPR)

luancarlosmkt@gmail.com - kovaleski@utfpr.edu.br - gaia@utfpr.edu.br - acfrancisco@utfpr.edu.br

Artigo submetido em setembro/2012 e aceito em fevereiro/2013

\section{RESUMO}

\begin{abstract}
A disponibilidade de dados de patentes em bancos nacionais e internacionais aumentou consideravelmente as possibilidades de análises destes documentos em diversos campos de pesquisa. Este trabalho objetivou analisar as informações tecnológicas contidas em bancos de patentes como estratégia na prospecção de novas tecnologias. Descreve as principais bases eletrônicas gratuitas, os documentos, a sua estrutura, códigos INID "Internationally agreed Numbers for the Identification of Data", indexação, referências, noções básicas de classificação. Além disto, analisa dados estatísticos das atividades do Centro de Divulgação, Documentação e Informação Tecnológica (CEDIN) do Instituto Nacional de Propriedade Industrial do Brasil (INPI) no período de 2000 a 2011. A pesquisa
\end{abstract}

demonstrou que as informações tecnológicas contidas em patentes podem ser colocadas para uma ampla faixa de utilização dentro das empresas e universidades. O CEDIN vem atendendo nos últimos anos usuários internos e externos para fornecimento e orientação nas bases de patentes e bibliográficas, porém este número ainda é pequeno, trabalhos de força-tarefa devem ser feitos entre o INPI, universidades e empresas, objetivando familiarizar potenciais usuários às informações tecnológicas. Contudo, esta ferramenta é indispensável para melhorar a pesquisa e desenvolvimento de produtos, processos e serviços nas empresas e universidades, pois as bases possibilitam quantidade, qualidade e acessibilidade das informações aos usuários.

PALAVRAS-CHAVE: Informação Tecnológica. Prospecção Tecnológica. Patentes. Propriedade Intelectual.

\section{TECHNOLOGICAL INFORMATION: IDENTIFYING TECHNOLOGIES, ADVANTAGES AND APPLICATIONS THROUGH THE NATIONAL AND INTERNATIONAL BANK OF PATENTS}

\begin{abstract}
The availability of patent data in national and international banks has greatly increased the possibilities for analysis of these documents in various fields of research. This study aims to analyze the technological information contained in patent databases as a strategy in the exploration of new technologies. It describes major free electronic databases, including their documents, structure, codes INID "Internationally agreed Numbers for the Identification of Data", index, references and basic classification. Moreover, it analyses statistical data of the activities of the Center for Technology Dissemination, Documentation and
\end{abstract}

Information (CEDIN) from the National Institute of Industrial Property of Brazil (INPI) in the period from 2000 to 2011 . The research showed that technological information contained in patents may be placed for a wide range of use within companies and universities. In recent yeras, CEDIN has been serving internal and external users by providing guidance on the basis of patents and literature, but this number is still small. In order to familiarize potential users of technological information, task forces should be gathered among INPI, universities and companies.

KEY-WORDS: Technological information. Technological Forecasting. Patents. Intellectual Property. 


\section{INFORMAÇÃO TECNOLÓGICA: IDENTIFICANDO TECNOLOGIAS, VANTAGENS E APLICAÇÕES ATRAVÉS DO BANCO NACIONAL E INTERNACIONAL DE PATENTES}

\section{INTRODUÇÃO}

Os economistas têm usado por muito tempo dados de patentes como um indicador de atividade inventiva e fonte de informação tecnológica para o setor produtivo e acadêmico. Eles testaram explicações e implicações do comportamento inventivo em indústrias e países, e têm caracterizado como "a natureza do ambiente tecnológico em que as empresas operam" (SCHMOOKLER, 1966; GLISMAN \& HORN, 1988; JAFFE, 1989).

Griliches (1990) revisou o fluxo na literatura, e verificou que os dados de patentes só estavam disponíveis de forma agregada, sendo necessário filtrar os documentos de patentes individuais. A disponibilidade das informações de patentes a partir de bancos internacionais já avançou consideravelmente, possibilitando analisar o comportamento de patenteamento nesses níveis.

O fácil acesso atualmente aos dados de patentes tem estimulado a pesquisa sobre as possibilidades e limitações do uso desses dados para analisar o desempenho tecnológico das empresas e também nas universidades.

Embora pudesse ser demonstrado que os dados de patentes são correlacionados como outro objetivo perceptual ou medidas da força tecnológica de empresas e universidades, isto não tem correlação efetiva com o desempenho financeiro (NARIN, NOMA \& PERRY, 1987).

No entanto, os trabalhos científicos deveriam apontar melhorias na medição do uso de informações, para além da contagem de patentes, bem como o desenvolvimento de técnicas analíticas destes documentos (BASBERG, 1987).

Segundo o World Intellectual Property Organization (WIPO), cerca de 2/3 das informações tecnológicas disponíveis em todo o mundo somente são reveladas nos documentos de patente (MARMOR, 1979). O acervo mundial de documentos está estimado em mais de 50 milhões com um crescimento anual da ordem de 1 milhão e 200 mil novos documentos (WIPO 2009).

Um estudo feito na Alemanha apontou que os custos de Pesquisa e Desenvolvimento (P\&D) poderiam ser minimizados em $30 \%$, caso a informação técnica disponível fosse utilizada pelas empresas e universidades. $O$ uso do conteúdo destes documentos é suficiente para que um profissional da área técnica da invenção consiga realizá-la de forma eficaz. Possui formato universal, dados bibliográficos com campos específicos numerados e contêm a informação mais recente em relação ao estado da técnica, coleções de documentos completos centralizadas em escritórios nacionais ou regionais de patentes, acesso por meios eletrônicos e abrange todos os campos tecnológicos.

Este trabalho teve como objetivo geral analisar as informações tecnológicas contidas em bancos de patentes como estratégia na prospecção de novas tecnologias. Descreve as principais bases internacionais, os documentos de patente, a sua estrutura, códigos INID "Internationally agreed Numbers for the Identification of Data", indexação, referências, noções básicas de classificação. Além disto, analisa dados estatísticos das atividades do Centro de Divulgação, Documentação e Informação Tecnológica (CEDIN) do Instituto Nacional de Propriedade Industrial do Brasil (INPI) no período de 2000 a 2011. 


\section{INFORMAÇÃO TECNOLÓGICA}

Conforme Parmagnani (2004) "a informação tecnológica é todo tipo de conhecimento sobre tecnologias de fabricação, de projeto e de gestão, que favoreça a melhoria contínua da qualidade e a inovação no setor produtivo".

Estas informações são encontradas em diversas fontes, sendo fontes informais obtidas em congressos, feiras e exposições, entrevistas em rádio, televisão etc. $E$ as fontes formais obtidas em livros, normas técnicas, teses, notícias em jornais e revistas, meio eletrônico (bases de dados nacionais ou internacionais, informações da internet, bibliotecas virtuais, artigos, patentes etc. (KAHANER, 1997).

O documento de patente é uma das mais ricas fontes de informação, pois o mesmo, por lei, deve possuir suficiência descritiva, conforme no Art. 24 (Lei de Propriedade Industrial, no 9.279/96). O relatório deverá descrever clara e suficientemente o objeto, de modo a possibilitar sua realização por técnico no assunto e indicar, quando for o caso, a melhor forma de execução.

Há dois tipos de documentos, o "pedido de patente", documento depositado, independente de ter sido concedido ou não, e a "patente concedida", documento final, após o processamento do pedido.

\subsection{Publicação do Documento de Patente}

No Brasil a publicação oficial sai na Revista da Propriedade Industrial (RPI), com dados bibliográficos e resumo. Parte da documentação disponível na íntegra via link para o Espacenet (2006 em diante) ou mediante solicitação de cópia do documento completo ao INPI, via correios.

Nos Estados Unidos até o ano 2000, apenas patentes concedidas, atualmente também para os pedidos. Confere nova numeração à patente concedida (ano da publicação na frente para o pedido depositado), e direito de não publicar pedidos não concedidos que não tenham sido depositados em outros países.

$\mathrm{Na}$ comunidade europeia, há pedidos e também patentes concedidas que mantêm o número, mas mudam o código de status do documento.

\subsection{Estrutura do Documento de Patente}

O documento de uma patente deve possuir informações bibliográficas (Folha de rosto), relatório descritivo, campo de reivindicações, desenhos quando necessário e resumo, conforme previsto em lei. Além disto, o documento possui numerações, chamadas códigos INID "Internationally agreed Numbers for the Identification of Data".

Estes códigos identificam todas as informações que constam da primeira página ou folha de rosto, tendo indicações de números, datas, origem, propriedade e técnica, como exemplo:

\subsection{Indicação dos números}

(11) Número do documento;

(21) Número designado ao documento quando de seu depósito;

(31) Número designado ao primeiro depósito (prioridade do documento).

\subsection{Indicação de datas}

(22) Data de depósito da solicitação; 
(32) Data de depósito da primeira solicitação (data de prioridade);

(41) a (47) Datas em que o pedido encontra-se disponível para ser visto;

(41) a (44) Referem-se a documentos de patentes publicados (examinados ou não, mas que ainda não têm a decisão final (concessão ou não da patente);

(45) a (47) Referem-se à data de concessão da patente, geralmente é utilizado o número (45).

\subsection{Identificação da origem/propriedade}

(19) Nome do país ou Organização Regional ou Internacional que publicou o documento de patente;

(33) País ou países do primeiro documento (prioridade);

(70) a (76) Identificação de partes relacionadas com o documento;

(71) Nome do depositante (quem recebeu a patente);

(72) Nome do inventor, se conhecido;

(73) Nome de quem detém os direitos sobre a patente;

(74) Nome do procurador ou agente;

(75) Nome do inventor, quando for também o depositante.

\subsection{Informação técnica}

(12) Indica o tipo de documento (patente de invenção, Modelo de Utilidade, etc);

(51) Classificação Internacional de Patente (IPC);

(52) Classificação Nacional ou doméstica de patente;

(54) Título da Invenção;

(56) Lista de documentos anteriores citados pelo depositante (pode auxiliar no exame) ou encontrados pelo examinador de patentes durante a busca para exame;

(57) Resumo do conteúdo do documento.

\subsection{Códigos do status da patente}

São códigos normatizados para a identificação dos diferentes tipos de documentos. No Brasil no final de 2008, o INPI introduziu códigos padronizados e começou a publicar também as patentes concedidas, conforme modelo abaixo:

\subsubsection{Pedidos de patente depositados:}

PI - Patente de invenção: ex. PI0900066-6 A2

C1 - Certificado de adição: ex. C10605141-3 E2

MU - Modelo de Utilidade: ex. MU8900022-6 U2

\subsubsection{Patentes Concedidas:}

PI - Patente de invenção: ex. PI5600001-2 B1

C1 - Certificado de adição: ex. C19605669-0 F1

MU - Modelo de utilidade: ex. MU8101158-0 Y1 


\subsubsection{Patentes Pipeline:}

PI11 - Patentes Pipeline concedidas: ex. PI1100008-2K B1

No Escritório Europeu de patentes, segue a seguinte padronização:

A1 - publicação do pedido de patente;

A2 - publicação do pedido sem o relatório de busca;

A3 - publicação do pedido com o relatório de busca;

B1 - publicação da patente concedida;

B2 - republicação da patente, por estar ilegível.

\subsection{Classificação Internacional de Patentes}

A Classificação Internacional de Patentes (CIP) teve suas primeiras discussões por volta de 1920. O texto da primeira edição da CIP foi estabelecido de acordo com a Convenção Europeia para a Classificação Internacional de Patentes, em 1954, tendo sua primeira edição em 1969 (WIPO 2009).

Entrou em vigor o Acordo de Estrasburgo, referente à CPI em 1974, que estabelece uma classificação comum para patentes de invenção. Qualquer país membro da Convenção de Paris pode se tornar membro do Acordo de Estrasburgo. A classificação é periodicamente revisada a partir de reuniões de peritos dos países membros da Organização Mundial da Propriedade Intelectual, sendo publicada em CD-Rom e podendo ser acessada nos sites do INPI e da OMPI.

Um dos objetivos da CPI foi criar uma ferramenta de busca e recuperação de documentos, sendo um instrumento para disposições organizadas destes documentos, a fim de facilitar o acesso às informações tecnológicas e legais contidas nos mesmos.

Além disto, é uma base de disseminação seletiva de informações a todos os usuários de patentes, base para investigar o estado da técnica em determinados campos da tecnologia, e base para preparar estatísticas sobre propriedade industrial que permitam a avaliação do desenvolvimento tecnológico em diversas áreas.

Até 1999 , eram reeditados a cada 5 anos. A oitava edição entrou em vigor no início de 2006, tendo modificações técnicas e de formato.

Atualmente, essa classificação divide os temas tecnológicos cobertos por patentes em mais de 64.000 itens, sendo compostas por 8 seções, 21 subseções, 120 classes, 628 subclasses, 69.000 grupos além de subgrupos, segundo os diversos setores industriais. As seções são as seguintes:
A - necessidades humanas correntes
B - técnicas industriais diversas, operações de processamento, transporte;
C - química e metalurgia;
D - têxteis e papel;
E - construções fixas;
F - engenharia mecânica, iluminação, aquecimento, armas e explosivos; 


$$
\begin{aligned}
& \text { G - física; } \\
& \text { H - eletricidade. }
\end{aligned}
$$

\subsection{Buscas nas principais Bases de Patentes Gratuitas}

Ao realizar buscas nas bases, objetiva-se alcançar vários parâmetros, dentre eles, a investigação prévia e/ou oficial de patenteabilidade, ações legais, pesquisa e desenvolvimento, e de interesses mercadológicos.

A principal limitação da busca ainda é a fase de sigilo (18 meses da data de depósito) dos documentos. Qualquer base de dados ou ferramenta de busca utilizada vai recuperar apenas documentos que já tenham sido publicados.

Existem os seguintes bancos e bases de dados:

1) bancos de patentes dos escritórios nacionais;

2) bases de dados em CD-Rom;

3) bases de dados eletrônicas gratuitas;

4) bases de dados eletrônicas comerciais.

Este trabalho teve como finalidade descrever somente as bases de dados eletrônicas gratuitas. Atualmente este tipo de base é a mais acessível, possui mecanismos de busca capazes de recuperar informações, usando diversos campos escolhidos dentro dos dados bibliográficos.

O Brasil foi o primeiro país do mundo a disponibilizar a sua base de patentes gratuitamente na rede.

As principais bases gratuitas são:

\subsection{World Intellectual Property Organization - WIPO}

Através do sistema Patentscope, esta base possibilita a busca e recuperação de informações relativas a depósitos de patentes PCT "Patent Cooperation Treaty" em vários campos.

A recuperação de dados é dada em uma listagem em formato xml, e também apresenta o resultado através de um conjunto de 4 gráficos, permitindo ao pesquisador ter uma visão instantânea da evolução do patenteamento no campo específico de pesquisa.

\subsection{Instituto Nacional de Propriedade Industrial - INPI}

Esta base disponibiliza informações institucionais pela internet, inclusive transferência de formulários e consulta à base de pedidos de patente nacionais, e a base de marcas.

É restrita a documentos depositados no âmbito doméstico e com data de publicação a partir de 1992, fornece somente os dados bibliográficos dos pedidos (folha de rosto digitalizada para alguns documentos em pdf).

Apresenta relatório de andamento dos processos, com os despachos já publicados. Além disto, realiza busca por diversos campos bibliográficos, e por palavras-chave em português.

O operador de truncagem de termos é o asterisco $(*)$, e possibilita utilizar operadores booleanos (and, or e and not). 


\subsection{European Patent Office - EPO}

Esta base permite a busca em patentes de vários países, desde 1970. O acesso a dados bibliográficos de documentos do escritório europeu e de mais de 80 países, e muitos documentos completos, disponibilizados em formato pdf, key words.

Permite acesso a até 500 registros encontrados por busca, após este número, somente via pagamento. Muitos documentos com relatório descritivo e reivindicações em formato HTML (facilita a localização dos termos buscados). Permite também o acesso à família de patentes dos documentos, apresentando o andamento do pedido nos países.

Possui 10 campos de busca específicos, até 10 termos de busca por campo, tendo os operadores booleanos (and, or e not). Os operadores de truncagem de termos são o asterisco (*), para número ilimitado de caracteres, a interrogação (?), para zero ou mais caracteres e o sustenido (\#), para somente um caracter.

\subsection{United States Patent and Trademark Office's - USPTO}

Esta base permite a busca em todas as patentes americanas concedidas desde 1790, tendo duas bases, a de patentes concedidas (1976, texto completo e 1790, textos digitalizados) e pedidos publicados (a partir de 03/2001).

Uma limitação que contém apenas documentos depositados ou publicados nos Estados Unidos. A busca pode ser feita no documento completo ou em campos específicos, e aceita um maior número de termos por campo.

Disponibiliza documentos disponíveis no formato original, possibilitando a recuperação de um panorama da tecnologia, através de documentos citados.

O operador de truncagem de termos é o cifrão (\$), e possibilita utilizar operadores booleanos (and, or e and not).

\section{METODOLOGIA}

Este estudo baseou-se em uma pesquisa bibliográfica, foram consultadas diversas fontes, tais como, livros, periódicos, manuais, leis, decretos, e sites. Do ponto de vista dos objetivos, caracterizou-se como exploratória e descritiva, pois buscou informações específicas do que foi desenvolvido (GIL, 2007).

No primeiro momento foi realizada uma definição e caracterização geral da informação tecnológica no contexto da propriedade industrial. No segundo momento, foram descritos elementos sobre a publicação de um documento de patente (sua estrutura, códigos INID "Internationally agreed Numbers for the Identification of Data", indexação, referências), e as noções básicas de Classificação Internacional de Patentes (CIP).

No terceiro momento, foram analisadas e descritas as principais bases eletrônicas gratuitas: Instituto Nacional da Propriedade Industrial (INPI), United States Patent and Trademark Office's (USPTO), World Intellectual Property Organization (WIPO) e European Patent Office (EPO).

A última etapa consistiu em analisar os dados estatísticos das atividades do Centro de Divulgação, Documentação e Informação Tecnológica (CEDIN) do INPI no período de 2000 a 2011. 
Estas informações foram coletadas diretamente no CEDIN em maio de 2012, para análise dos dados, foi utilizada estatística descritiva, e os dados foram tabulados no programa Microsoft Excel 2007.

\section{ANÁLISE DOS RESULTADOS}

O Centro de Divulgação, Documentação e Informação Tecnológica (CEDIN), teve sua implantação em 1976, compreendendo o Banco de Patentes e a Biblioteca do INPI. Conforme no Art.13 (Decreto 77483/76), suas atribuições consistem em proporcionar às áreas interessadas, privadas e governamentais, informações sobre o desenvolvimento tecnológico industrial, em âmbito nacional e internacional.

O CEDIN é especializado em Propriedade Industrial, possui em seu acervo legislações e publicações de vários Escritórios Internacionais de Patentes, assim como livros e revistas técnicas, atendendo a usuários internos e externos.

A figura 1 ilustra a evolução sobre o fornecimento de cópias de documentos de patentes e de material bibliográfico pelo CEDIN nos últimos 11 anos para os clientes internos e externos.

Nota-se que o fornecimento de documento de patente é muito superior ao de material bibliográfico, chegando a quase 92 mil documentos fornecidos em 2008, tendo uma considerável evolução nos últimos anos. Esta discrepância se deve ao fato de o documento de patente ser considerado como conhecimento aplicado, contendo muito mais informações técnicas do que o material bibliográfico, considerado conhecimento básico.

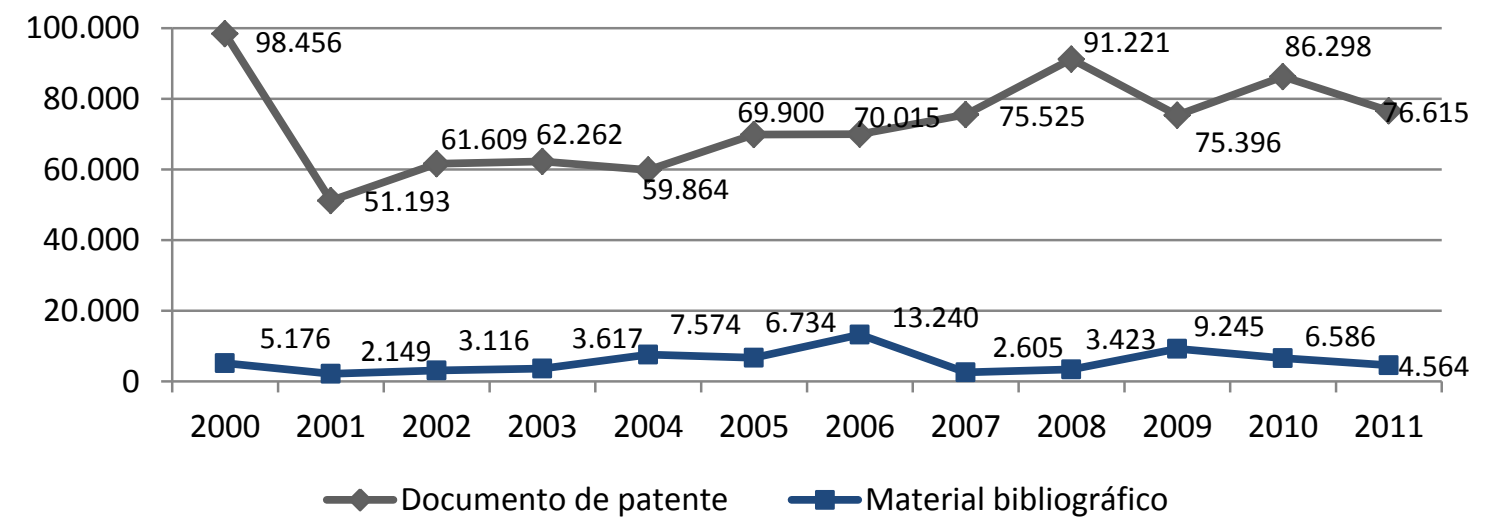

Figura 1: Fornecimento de cópia de documentos de patentes e de material bibliográfico Fonte: CEDIN (2012)

A figura 2 detalha sobre os atendimentos e orientações realizados pelo CEDIN em bases de patentes e bibliográficas.

As bases de patentes sempre tiveram um número maior de solicitações, mas a partir de 2006 as bases bibliográficas também cresceram consideravelmente. 


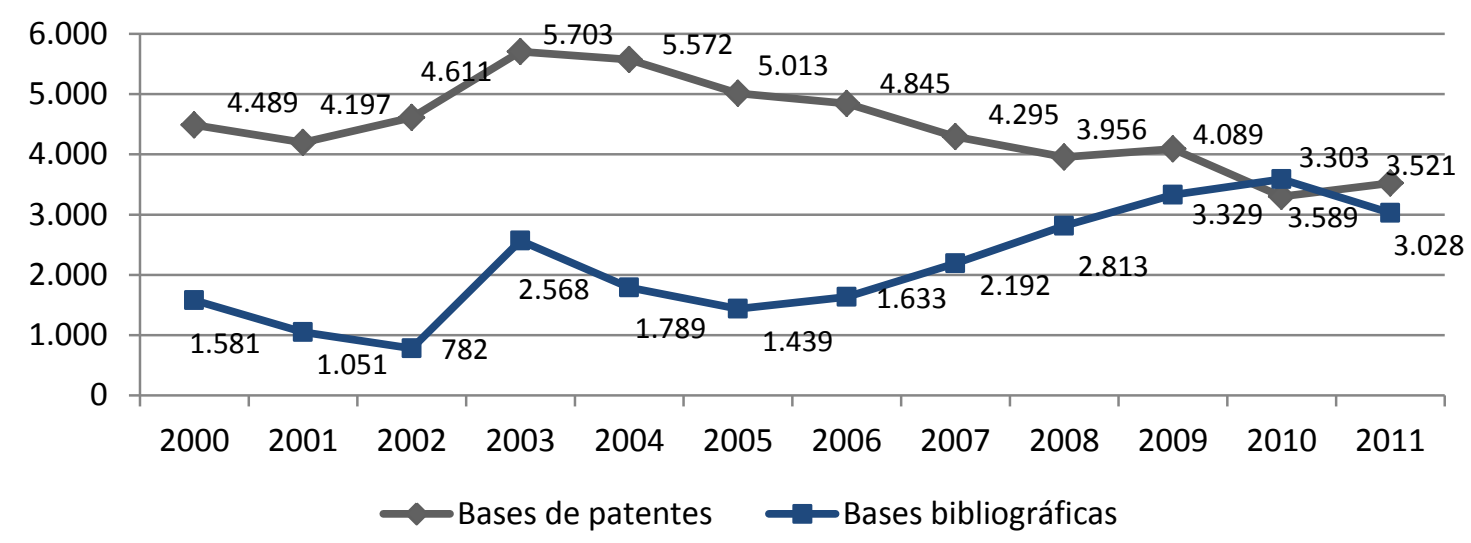

Figura 2: Atendimento e Orientação aos Usuários Fonte: CEDIN (2012)

A figura 3 ilustra as buscas realizadas pelo CEDIN e usuários, bem como as buscas bibliográficas.

Observa-se que as buscas realizadas pelos usuários sempre se manteve superior às realizadas pelo CEDIN, apenas em 2010 e 2011 que este indicador foi quase igualado.

Um dado interessante que podemos extrair desta estatística é, se considerarmos o alto número de invenções publicadas, desperdícios de verba com pesquisa e desenvolvimento, insuficiência descritiva em muitos pedidos de patentes que temos aqui no Brasil, com as buscas de informações tecnológicas feitas pelos próprios usuários, que em muitos casos não estão familiarizados com estas ferramentas.

A falta de orientação de especialistas para busca de anterioridade estabelece uma margem maior a erros, podendo acarretar o arquivamento definitivo do pedido.

A busca bibliográfica contém apenas resultados dos últimos dois anos, pois este indicador foi utilizado a partir de 2010.

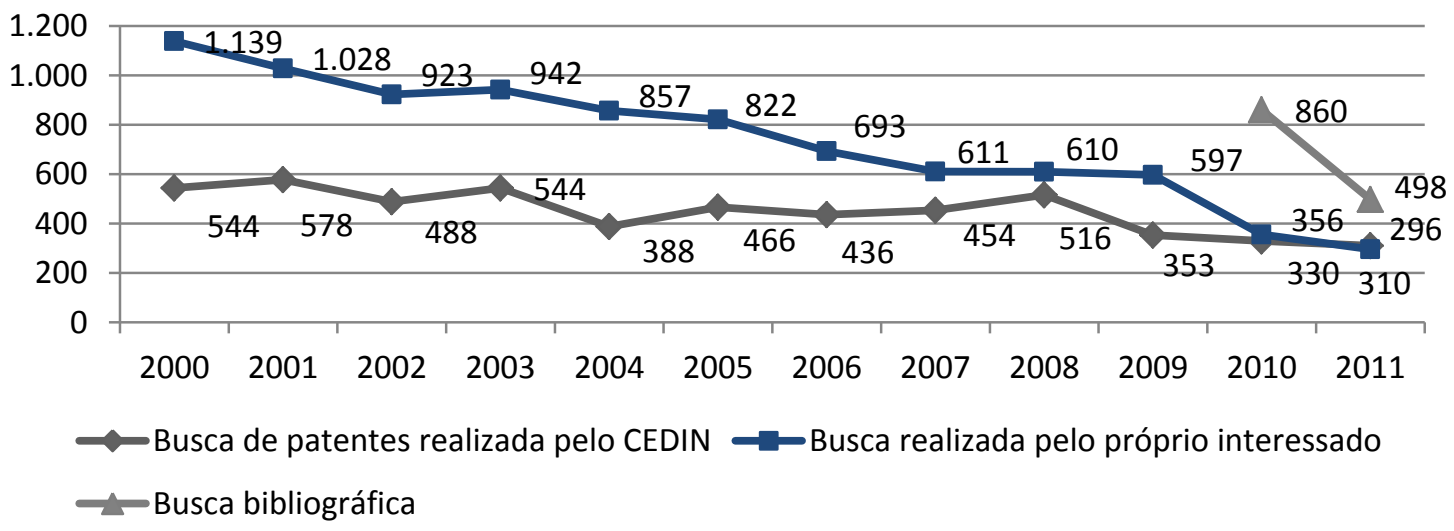

Figura 3: Buscas pelo CEDIN e usuários

Fonte: CEDIN (2012) 
Por último, a figura 4 fornece informações sobre o número de empresas cadastradas no Programa de Fornecimento Automático de Informação Tecnológica (Profint), que nos últimos 7 anos tiveram uma diminuição considerável de novos cadastramentos.

Este programa faz parte do CEDIN, e tem como objetivo geral disseminar seletivamente a informação tecnológica contida em documentos de patentes, nacionais e internacionais, sendo este serviço de fundamental importância para as empresas.

Uma vez cadastradas, as empresas recebem as documentações nas áreas de seu interesse, à medida que a documentação é incorporada ao banco de patentes, de forma regular e automática.

Uma das vantagens mais importantes é que mantém a empresa atualizada com os avanços tecnológicos de sua área de atuação e permite o monitoramento das atividades tecnológicas dos concorrentes, no Brasil e em outros países.

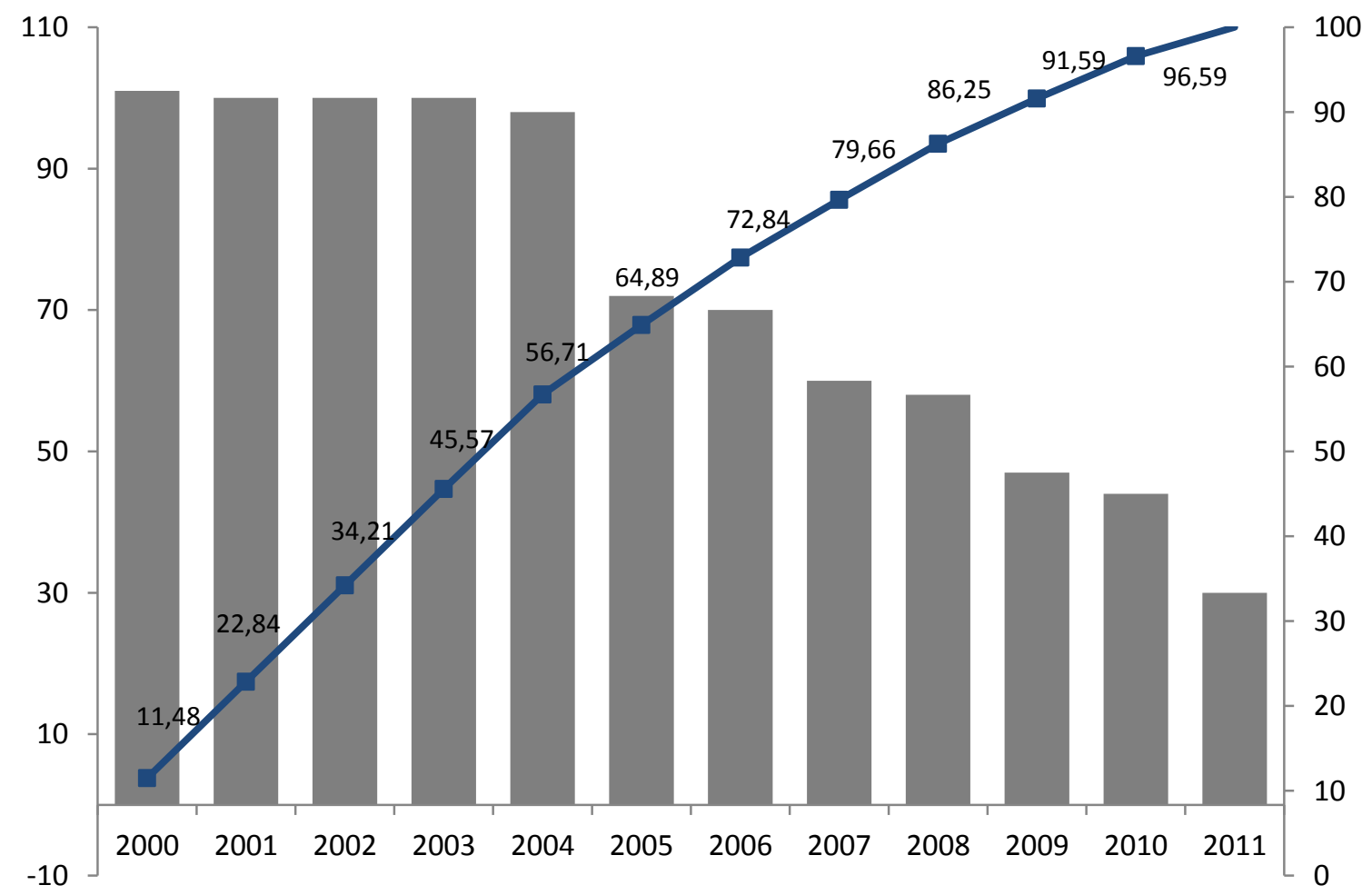

Figura 4: Número de Empresas do Profint Fonte: CEDIN (2011)

\section{CONCLUSÃO}

Este trabalho demonstrou que as informações tecnológicas contidas em patentes podem ser colocadas para uma ampla faixa de utilização dentro das empresas e universidades, pois existem muitas vantagens em seu uso efetivo, dentre delas:

- Definir o estado da técnica de determinada tecnologia visando o depósito de uma patente; 
- Fundamentar decisões de investimento (melhores condições de compra de tecnologia); Possibilitar conhecer potenciais alternativas técnicas, identificar tecnologias emergentes, tendências de mercado e previsão de novos produtos.

- Definir potenciais rotas para aperfeiçoamentos em produtos e processos existentes, monitorar as atividades dos concorrentes.

- Analisar a validade (verificar se a tecnologia está disponível no país, evitando processos judiciais), evitar contrafação.

- Mapear citações em patentes, o que permite o rastreamento de tecnologias.

- Possibilitar o levantamento das tecnologias em nível mundial por empresa, inventor, assunto.

- Analisar as famílias de patentes (permite verificar os países onde se busca proteção para uma mesma invenção) e por último, possibilitar o subsídio ao exame técnico ou nulidade administrativa.

O CEDIN vem atendendo nos últimos anos a usuários internos e externos para fornecimento e orientação nas bases de patentes e bibliográficas, porém este número ainda é pequeno, trabalhos de força-tarefa devem ser feitas entre o INPI, universidades e empresas, objetivando familiarizar potenciais usuários às informações tecnológicas contidas em patentes.

Um trabalho similar deve ser feito também no Programa de Fornecimento Automático de Informação Tecnológica (Profint), pois nos últimos sete anos teve uma diminuição considerável de novos cadastramentos.

Contudo, esta ferramenta é indispensável para melhorar a pesquisa e desenvolvimento (P\&D) de produtos, processos e serviços nas empresas e universidades, pois as bases possibilitam quantidade, qualidade e acessibilidade das informações aos usuários.

\section{AGRADECIMENTOS}

Agradecemos ao Conselho Nacional de Desenvolvimento Científico e Tecnológico - CNPq pelo financiamento da pesquisa.

\section{REFERÊNCIAS BIBLIOGRÁFICAS}

1. BASBERG, B. L. Patents and the measurement of technological change: a survey of the literature. Research Policy. 16: 131-141, 1987.

2. BRASIL. Lei de Propriedade Intelectual do Brasil no 9.279, de 14 de maio de 1996. Disponível em: < http://www.planalto.gov.br/ccivil_03/leis/L9279.htm>. Acesso em: 26 mar 2011.

3. EPO, European Patent Office. Disponível em:<www.epo.org/>. Acesso em maio de 2012.

4. GIL, A. C. Como elaborar projetos de pesquisa. 1ạ ed, São Paulo: Atlas, 2007.

5. GLISMAN, H. H; HORN, E.-J. Comparative invention performance of major industrial countries: patterns and explanations. Management Science. 34: 1169-I 187, 1988. 
6. GRILICHES, Z. Patent statistics as economic indicators: survey. Journal of Economic Literature. 28: 1661-1707, 1990.

7. Guia da Classificação Internacional de Patentes: Organização Mundial da Propriedade Intelectual, 8a ed. Genebra, 2006.

8. INPI, Instituto Nacional de Propriedade Intelectual. Dados Estatísticos do Centro de Divulgação, Documentação e Informação Tecnológica - CEDIN. Disponível em:< www.inpi.gov.br/ > Acesso em maio de 2012.

9. JAFFE, A, B. Characterizing the "technological position" of firms. with application to quantifying technological opportunity and research spillovers. Research Policy. 18: 87-97, 1989.

10. KAHANER, L. Competitive intelligence: how to gather, analyze, and use information to move your business to the top. New York: Touchstone, 1997.

11. MARMOR, A. C. et al. The Technology assessment and forecast program of the United States Patent in Trademark Office. World Patent Information, Munchen, v.1. n.1. p.15-23, 1979.

12. NARIN, F.; NOMA, E.; PERRY, R. Patents as indicators of corporate technological strength. Research Policy. 16: 143-155, 1987.

13. PARMAGNANI, R. M. O Glossário Geral de Ciência da Informação. Faculdade de Ciência da Informação (FCl) da Universidade de Brasília. 2004, Disponível em: < http://www.cid.unb.br/publico/setores/100/123/sistema/m0039015.htm>. Acesso em 15/04/2012.

14. SCHMOOKLER, J. Invention and Economic Growth. Harvard University Press. Cambridge. 1966.

15. USPTO, United States Patent and Trademark Office's. Disponível em:< www.uspto.gov/>. Acesso em maio de 2012.

16. WIPO, World Intellectual Property Organization. Disponível em:<www.wipo.int $>$. Acesso em maio de 2012. 